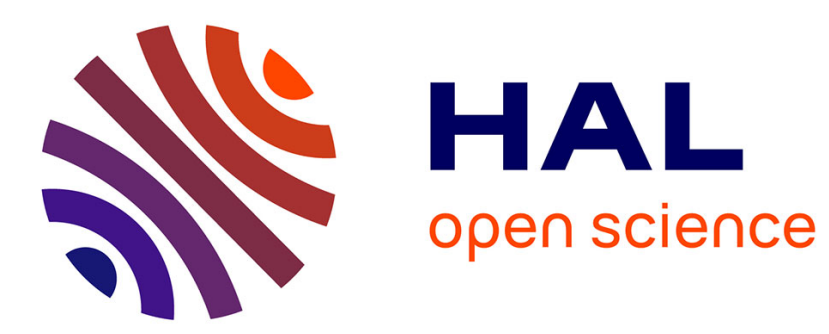

\title{
Shear strength and force transmission in granular media with rolling resistance
}

Nicolas Estrada, Alfredo Taboada, Farhang Radjai

\section{To cite this version:}

Nicolas Estrada, Alfredo Taboada, Farhang Radjai. Shear strength and force transmission in granular media with rolling resistance. Physical Review E: Statistical, Nonlinear, and Soft Matter Physics, 2008, 78 (2), pp.021301. 10.1103/PhysRevE.78.021301 . hal-00412020

\section{HAL Id: hal-00412020 \\ https://hal.science/hal-00412020}

Submitted on 26 Apr 2021

HAL is a multi-disciplinary open access archive for the deposit and dissemination of scientific research documents, whether they are published or not. The documents may come from teaching and research institutions in France or abroad, or from public or private research centers.
L'archive ouverte pluridisciplinaire HAL, est destinée au dépôt et à la diffusion de documents scientifiques de niveau recherche, publiés ou non, émanant des établissements d'enseignement et de recherche français ou étrangers, des laboratoires publics ou privés. 


\title{
Shear strength and force transmission in granular media with rolling resistance
}

\author{
Nicolas Estrada* and Alfredo Taboada \\ Géosciences Montpellier, Université de Montpellier II and CNRS, cc060, Place Eugène Bataillon, 34095 Montpellier cédex 5, France \\ Farhang Radjaï \\ LMGC, Université de Montpellier II and CNRS, cc048, Place Eugène Bataillon, 34095 Montpellier cédex 5, France
}

(Received 8 May 2008; published 1 August 2008)

\begin{abstract}
We investigate a class of granular materials characterized by the possibility of interlocking between the particles. The interlocking is modeled by its effect through rolling resistance depending on relative rotation and normal force at the contact points and involving a single parameter analogous to the sliding friction coefficient. The model, which is introduced in the framework of the contact dynamics method, is applied to simulate the simple shear of a large granular sample. We present a detailed analysis regarding the influence of rolling and sliding friction parameters on the macroscopic response in terms of shear strength, fabric properties, and force transmission. Interestingly, two distinct regimes can be distinguished in which the steady-state shear strength is controlled by either rolling resistance or sliding friction. The relative contributions of rolling and sliding contacts to the shear strength are consistent with the same two regimes. Interlocking strongly affects the force network by enhancing the arching effect and thus increasing the relative importance of weak contact forces and torques, which is reflected in a decreasing power-law probability distribution of the contact forces and torques below the mean. Due to the combined effect of friction and interlocking, the force-carrying backbone takes an increasingly columnar aspect involving a low fraction of particles. Our data suggest that the nature of the weak contact network is strongly affected by the formation of these columns of particles which do not need to be propped laterally. In particular, in the limit of high rolling resistance and sliding friction, the role of the weak network of contacts is no longer to prop the force chains, but, like the strong contact network, to actively sustain the deviatoric load imposed on the system.
\end{abstract}

DOI: 10.1103/PhysRevE.78.021301

PACS number(s): $83.80 . \mathrm{Fg}, 81.05 . \mathrm{Rm}, 45.70 . \mathrm{Mg}$

\section{INTRODUCTION}

Granular materials are characterized by a shear strength depending on both the stress level and density. As a result of the quasirigidity of the grains and Coulomb friction, the maximum shear stress $\tau$ on a slip plane varies proportionally to the normal stress $\sigma$, so that the strength is generally described by the ratio $\mu=\tau / \sigma$ which depends only on the solid fraction. Only in a steady state reached after sufficiently long shearing can, this ratio be considered as a material property. This state, often called the "critical state" in soil mechanics, is thus characterized by a critical solid fraction $\nu$ and a (normalized) shear strength $\mu^{*}$, also described as the coefficient of internal friction of the granular material [1,2].

Unlike solid friction, which is a surface property, the shear strength $\mu^{*}$ represents a bulk property resulting from a collective process, which depends on the nature of grain interactions and their geometrical arrangement. However, the link between shear strength and grain interactions is still a largely open issue. Early models of shear strength in granular media were based on the assumption that the dominant mechanism of failure and dissipation at the contact scale is sliding. These models predicted that $\mu^{*}$ increased indefinitely with the coefficient of sliding friction $\mu_{s}$ between the grains [3-5]. However, it was later found, both experimentally and theoretically, that $\mu^{*}$ increases indeed with $\mu_{s}$ but saturates rapidly to a limit value that is independent of $\mu_{s}$

\footnotetext{
*estrada@gm.univ-montp2.fr
}

$[6,7]$. This behavior highlights the fact that, even though sliding friction is the dominant source of dissipation in plastic flow of granular materials, the major grain-scale mode of deformation is the rolling of grains over each other which keeps $\mu^{*}$ at a low level. Recent numerical simulations indicate that, in granular media composed of spherical grains, sliding occurs only at a small proportion $(<10 \%)$ of contacts, and these sliding contacts are basically those carrying weak normal forces $[8,9]$.

These observations suggest that the shear strength should be strongly affected when the relative rolling is restrained by a mechanism of interlocking between the grains. Weak rolling resistance can result from interactions between grain surface irregularities or asperities. Larger rolling resistance may be due to interlocking between grains of nonconvex or angular shapes often occurring in geomaterials (gouge material, ballast, etc.) or in materials with dendritic grain surface structures (flake-shaped metallic powder, snowflakes, etc.). The presence of a cementing material between grains can be another cause of interlocking. In all cases, independently of the underlying mechanisms, interlocking implies rolling resistance between the grains.

In order to be able to simulate real materials by means of discrete element methods, several authors have specifically incorporated rolling resistance at the level of contact interactions between grains [10-16]. Other authors have simply hindered grain rotations. This approach can be a useful trick to achieve very high values of $\mu^{*}$ whilst using spherical-shape particles [17-20]. Even though the practical interest of introducing rolling resistance is evident, the joint effect of sliding friction and rolling resistance on the macroscopic behavior 
of granular materials has not been systematically investigated.

In this paper, we use a model of interlocking in the framework of the contact dynamics method in order to study the steady-state strength properties and microstructure of sheared granular media. In our model, the interlocking between grains is introduced through a rolling friction law relating the relative rotation of the grains with a contact torque which is proportional to the normal force. This law involves thus a rolling friction coefficient $\mu_{r}$ analogous to the sliding friction coefficient $\mu_{s}$. Polydisperse two-dimensional (2D) granular samples were sheared under simple shear boundary conditions and analyzed in the steady state for a broad range of friction parameters $\mu_{s}$ and $\mu_{r}$ in terms of shear strength, fabric properties and force transmission. An interesting finding of this work is that the saturation of $\mu^{*}$ occurs also as a function of $\mu_{r}$ with a saturation value that increases with $\mu_{s}$, and, symmetrically, as a function of $\mu_{s}$ with a saturation value increasing with $\mu_{r}$. As a result, two distinct regimes can be distinguished in which $\mu^{*}$ is controlled by either $\mu_{s}$ or $\mu_{r}$. We consider in detail the effect of interlocking on the microstructure and force transmission in these two regimes.

The outline of this paper is as follows. We first describe, in Sec. II, the numerical method, system characteristics, and loading parameters. In Sec. III, we present a parametric study of the shear strength as a function of the two friction coefficients. In Sec. IV, we focus on the solid fraction and structure of the force-carrying backbone. Section $\mathrm{V}$ is devoted to the force distributions and the influence of rolling friction on the bimodal behavior of our granular system. We conclude, in Sec. VI, with a summary of the main results and a brief discussion about the extensions of this work.

\section{MODEL DESCRIPTION}

\section{A. Contact dynamics method}

The simulations were carried out using the contact dynamics method, which is suitable for simulating large assemblies of rigid particles [21-24]. This method is based on a nonsmooth formulation of the equations of motion in which the accelerations are replaced by possible velocity jumps resulting from collisions. The frictional and collisional interactions are described as "complementarity relations" between the relative velocities and the corresponding forces at the contact points.

In the normal direction, the condition of impenetrability of the grains at a contact, i.e., when the gap $\delta=0$, implies the following complementary relation:

$$
\begin{aligned}
& u_{n}=0 \Rightarrow f_{n} \geqslant 0, \\
& u_{n}>0 \Rightarrow f_{n}=0,
\end{aligned}
$$

where $u_{n}$ is the relative normal velocity and $f_{n}$ is the normal contact force. This relation, called Signorini's conditions for velocities, is shown as a graph in Fig. 1(a). Remark that this relation cannot be reduced to a (mono)valued functional dependence between the two variables. In the same way, in the tangential direction, the Coulomb friction law can be de- (a)
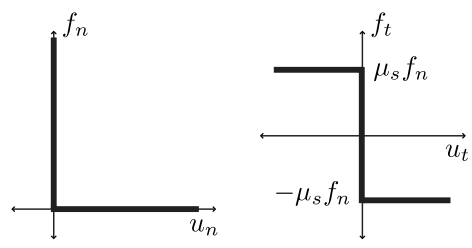

FIG. 1. (a) Signorini's conditions relating the normal force $f_{n}$ and the relative normal velocity $u_{n}$. (b) Coulomb friction law relating the tangential force $f_{t}$ and the relative tangential veolcity $u_{t}$.

scribed by the following complementary relation:

$$
\begin{gathered}
u_{t}>0 \Rightarrow f_{t}=-\mu_{s} f_{n}, \\
u_{t}=0 \Rightarrow-\mu_{s} f_{n} \leqslant f_{t} \leqslant \mu_{s} f_{n}, \\
u_{t}<0 \Rightarrow f_{t}=\mu_{s} f_{n},
\end{gathered}
$$

where $u_{t}$ is the sliding velocity and $f_{t}$ is the friction force. Figure 1(b) displays Coulomb's conditions as a graph. Like Signorini's relation, this is a nonsmooth contact law in the sense that it is a set-valued function.

Both contact laws are implemented within the contact dynamics method without introducing further regularizing or damping parameters. The formulation of the contact laws at the velocity level implies an implicit time-stepping scheme and the determination of the forces and velocities at each time step through an iterative procedure similar to a GaussSeidel scheme [21,23-27].

\section{B. Rolling resistance}

In order to introduce interlocking between grains, the idea is to replace the effect of grain shape (nonconvex or angular shapes) by a contact law allowing for rolling resistance. The local kinematic variable is the relative angular velocity $\omega_{r}$ at the contacts between grains. For two disks with angular velocities $\omega^{i}$ and $\omega^{j}$, we have

$$
\omega_{r}^{i j}=\omega^{i}-\omega^{j} .
$$

Interlocking between the grains $i$ and $j$ corresponds to $\omega_{r}^{i j}$ $=0$. From a dynamic point of view, this condition requires the transmission of a torque $M$ at the contact point. This is analogous to the nonsliding condition $u_{t}=0$ which requires the activation of the friction force $f_{t}$ (vertical branch of Coulomb's law in Fig. 1). In the same way, the rolling resistance corresponds to a threshold $M_{c}$ on the contact torque. Hence, by analogy with Coulomb's friction law, we introduce the following complementarity relation between $\omega_{r}$ and $M$ :

$$
\begin{gathered}
\omega_{r}>0 \Rightarrow M=-M_{c}, \\
\omega_{r}=0 \Rightarrow-M_{c} \leqslant M \leqslant M_{c}, \\
\omega_{r}<0 \Rightarrow M=M_{c} .
\end{gathered}
$$

To push this analogy further, let us compare the force balance for a block and a disk of radius $R$ placed on an 
(a)

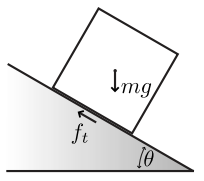

(b)

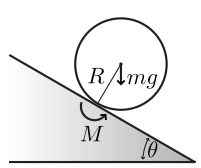

FIG. 2. Analogy between a block (a) and a disk of radius $R$ (b) placed on an inclined plane. The stability of the block is ensured by the friction force $f_{t}$; the stability of the disk, with respect to rolling, is ensured by the contact moment $M$.

inclined plane; see Fig. 2. The stability of the block is due to the friction force $f_{t}=m g \sin \theta<\mu_{s} m g \cos \theta$. Sliding occurs for $\theta=\phi_{s}$, for which $f_{t}=\mu_{s} f_{n}$. The stability of the disk, with respect to rolling, can only be ensured by a contact torque $M$ balancing exactly the moment of its weight about the contact point $M=m g R \sin \theta<M_{c}$. Assuming that, as for sliding, rolling occurs for an inclination $\theta=\phi_{r}$ of the plane, we get $M_{c}$ $=\mu_{r} R m g \cos \phi_{r}=\mu_{r} R f_{n}$, where $\mu_{r}=\tan \phi_{r}$ is the coefficient of rolling friction. With this choice, the complementarity relation defining the rolling friction law between two grains of radii $R_{i}$ and $R_{j}$ becomes

$$
\begin{gathered}
\omega_{r}>0 \Rightarrow M=-\mu_{r} \ell f_{n}, \\
\omega_{r}=0 \Rightarrow-\mu_{r} \ell f_{n} \leqslant M \leqslant \mu_{r} \ell f_{n}, \\
\omega_{r}<0 \Rightarrow M=\mu_{r} \ell f_{n},
\end{gathered}
$$

where $\ell=R_{i}+R_{j}$. The graph of this relation is displayed in Fig. 3.

The scaling of the rolling threshold $M_{c}$ with $\ell$ leads to comparable values of $\mu_{r}$ and $\mu_{s}$. In the example of the inclined plane illustrated in Fig. $2, \mu_{s}=\mu_{r}$ implies that sliding of the block and rolling of the disk begin for the same inclination angle $\phi_{s}=\phi_{r}$.

Although this model is basically meant to isolate the effect of moment transfer between particles, a relevant question is whether a rolling resistance proportional to the normal force, as in relation (5), can be identified with a real physical mechanism in a noncohesive material. As weak rolling resistance results from micro slidings between the asperities,

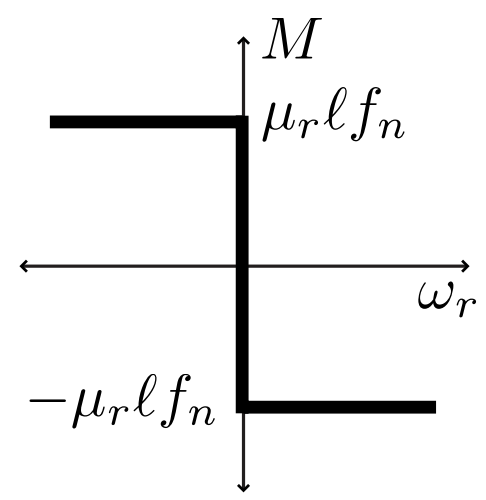

FIG. 3. Complementary relations defining the rolling friction law. $M$ is the torque and $\omega_{r}$ is the relative angular velocity at the contacts between grains.

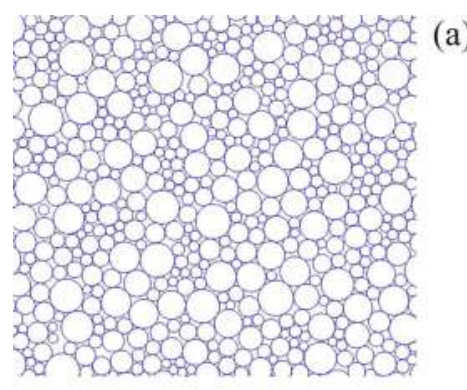

(a)

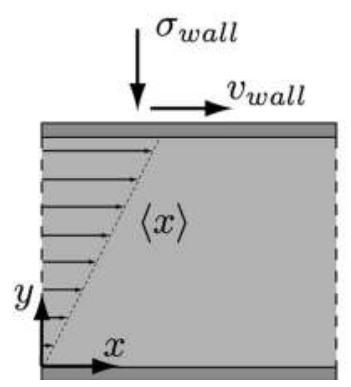

(b)

FIG. 4. (Color online) (a) Snapshot of a portion of the packing before shearing. (b) Schematic representation of the simple shear test; the dashed lines represent periodic boundaries. $v_{\text {wall }}$ is the horizontal velocity of the wall, $\sigma_{\text {wall }}$ is the confining pressure, and $\langle x\rangle$ is the averaged horizontal displacement of the particles

large rolling resistance can stem from a similar mechanism at a larger scale, e.g., between particles with dendritic surfaces. Indeed, strongly angular and nonconvex particles can interlock and form several contact points, each resisting to sliding through a Coulomb friction. The order of magnitude of $M_{c}$ is then proportional to the mean normal force $f_{n}$ between the two particles, the coefficient of sliding friction at individual contact points, and the typical separation distance between these contact points.

We implemented the above rolling friction law in the framework of the contact dynamics method. Below, we investigate in detail the influence of the two friction parameters on the shear strength and force transmission.

\section{Simple shear test}

Our numerical samples are composed of 7500 disks with diameters uniformly distributed by volume fractions between $0.6\langle d\rangle$ and $2.4\langle d\rangle$, where $\langle d\rangle$ is the mean diameter. The particles are initially placed in a semiperiodic box $100\langle d\rangle$ wide, using a geometrical procedure [26,28]; see Fig. 4(a). Next, the packing is sheared by imposing to the upper wall a constant horizontal velocity $v_{\text {wall }}$ and a constant pressure $\sigma_{\text {wall }}$; see Fig. 4(b). To avoid strain localization at the boundaries, sliding and rolling are inhibited for the particles in contact with the walls. The gravity is set to zero.

Since we are interested in the steady state behavior, the samples are sheared and maintained in the steady state up to a large cumulative shear strain $\gamma=x_{\text {wall }} / y_{\text {wall }}=5$, where $x_{\text {wall }}$ is the horizontal displacement of the upper wall and $y_{\text {wall }}$ is its vertical position. Figures 5(a) and 5(b) show the shear ratio $\tau_{\text {wall }} / \sigma_{\text {wall }}$, where $\tau_{\text {wall }}$ is the tangential stress at the moving wall, and the normalized volume of the packing 

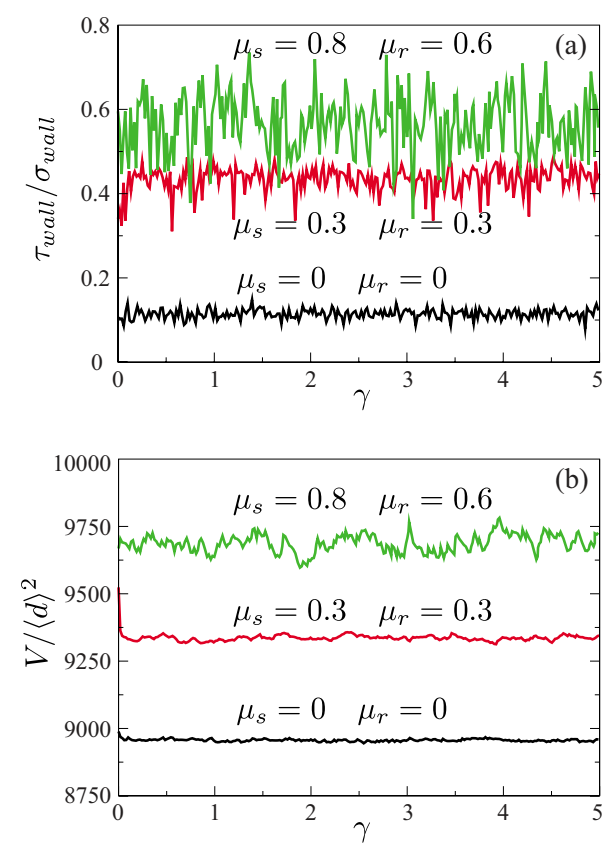

FIG. 5. (Color online) (a) Shear ratio $\tau_{\text {wall }} / \sigma_{\text {wall }}$, and (b) normalized volume of the packing $V /\langle d\rangle^{2}$, as functions of the shear strain $\gamma$.

$V /\langle d\rangle^{2}$ as a function of the shear strain $\gamma$ for three different values of $\mu_{s}$ and $\mu_{r}$. The packing is at the steady state since $\tau_{\text {wall }} / \sigma_{\text {wall }}$ and $V /\langle d\rangle^{2}$ fluctuate around a mean value. Figure 6 shows the profiles of the averaged horizontal displacement $\langle x\rangle$ of the particles versus their vertical position $y$ for five extreme values of $\mu_{s}$ and $\mu_{r}$. These profiles are almost linear, showing that the shear strain is uniform in the bulk.

We may also evaluate the average level of inertia in our simple shear tests by evaluating the inertia parameter $I$ defined as [29-31]

$$
I=\frac{\dot{\gamma}\langle d\rangle}{\sqrt{\sigma_{\text {wall }} / \rho}},
$$

where $\dot{\gamma}$ is the shear rate, $\langle d\rangle$ is the average grain size, and $\rho$ is the density. A sheared system is in a quasistatic state if $I$

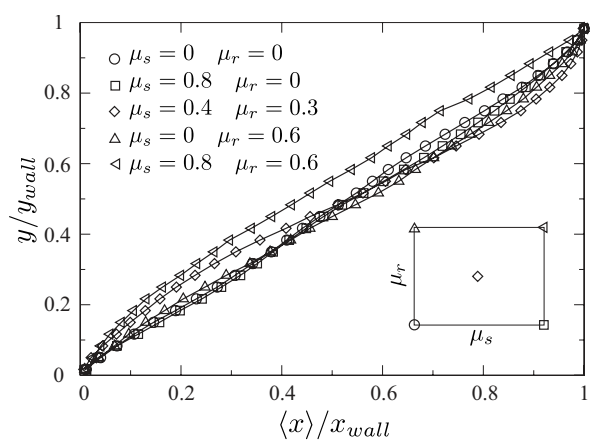

FIG. 6. Profiles of the normalized averaged horizontal displacement of the particles $\langle x\rangle / x_{\text {wall }}$, versus their normalized vertical position in the sample $y / y_{\text {wall }}$, at the end of the shear test, for five extreme combinations (see inset) of the two friction coefficients $\mu_{s}$ and $\mu_{r}$. $\ll 1$. In all our tests we have $\dot{\gamma}=1 \times 10^{-6} / \Delta t$ and $\sigma_{\text {wall }}=1$ $\times 10^{-4} \rho(\langle d\rangle / \Delta)^{2}$. Hence, $I \simeq 10^{-4}$, which means that our sheared samples can reasonably be considered to be in a quasistatic state.

\section{SHEAR STRENGTH}

The steady-state shear strength of a frictional granular material is characterized by the coefficient of internal friction $\mu^{*}$, which should be calculated from an estimate of the stress tensor $\boldsymbol{\sigma}$. The latter can be calculated at any stage of deformation from the simulation data which give access to the contact network and forces. We first compute the internal moment tensor $\boldsymbol{M}^{p}$ for each grain defined by $[32,33]$

$$
M_{\alpha \beta}^{p}=\sum_{c \in p} f_{\alpha}^{c} r_{\beta}^{c},
$$

where $\alpha$ and $\beta$ represent the components in an orthonormal reference frame, $f^{c}$ is the force exerted on particle $p$ at contact $c$, and $\boldsymbol{r}^{c}$ is the position vector of the same contact. The summation runs over all the contacts $c$ of particle $p$. The average stress tensor $\boldsymbol{\sigma}$ in the volume $V$ of the granular assembly is given by

$$
\sigma_{\alpha \beta}=\frac{1}{V} \sum_{p \in V} M_{\alpha \beta}^{p} .
$$

This expression allows us to calculate the vertical normal stress $\sigma_{y y}$ and the tangential stress $\tau=\sigma_{x y} \cong \sigma_{y x}$ in the steady state, and thus the coefficient of internal friction which in simple shear is

$$
\mu^{*}=\frac{\tau}{\sigma_{y y}} .
$$

The dissipation rate in the steady state is simply given by $W=\mu^{*} \dot{\gamma} \sigma_{y y}$.

We performed a large number of shear simulations for a broad set of combinations of the friction coefficients. The sliding friction coefficient $\mu_{s}$ was varied from 0 to 0.8 by steps of 0.05 , and, for each value of $\mu_{s}$, the rolling friction coefficient $\mu_{r}$ was varied from 0 to 0.6 by steps of 0.05 . Hence, a total number of 221 simulations, each for a total cumulative shear strain of 5, were performed. For each combination, the average value of $\mu^{*}$ was taken over the last $60 \%$ of the cumulative shear strain in order to get a representative steady-state value of $\mu^{*}$.

Figure 7(a) shows $\mu^{*}$ as a function of $\mu_{s}$ for several fixed values of $\mu_{r}$. We see that, for each value of $\mu_{r}$, the coefficient of internal friction $\mu^{*}$ increases as a function of $\mu_{s}$ and saturates after a short transient at a saturation value that increases with $\mu_{r}$. This result generalizes a behavior previously established in the case $\mu_{r}=0$ [26]. Symmetrically, as shown in Fig. 7(b), for each value of $\mu_{s}, \mu^{*}$ increases and saturates as a function of $\mu_{r}$. These data also show that the internal friction coefficient in the absence of rolling resistance $\left(\mu_{r}=0\right)$ cannot exceed 0.3 . On the other hand, it takes a constant value of 0.15 with rolling resistance in the absence of sliding friction $\left(\mu_{s}=0\right)$.

The contour map of $\mu^{*}$ is represented in Fig. 8 as a function of $\mu_{s}$ and $\mu_{r}$ for the whole set of our shear simulations. 

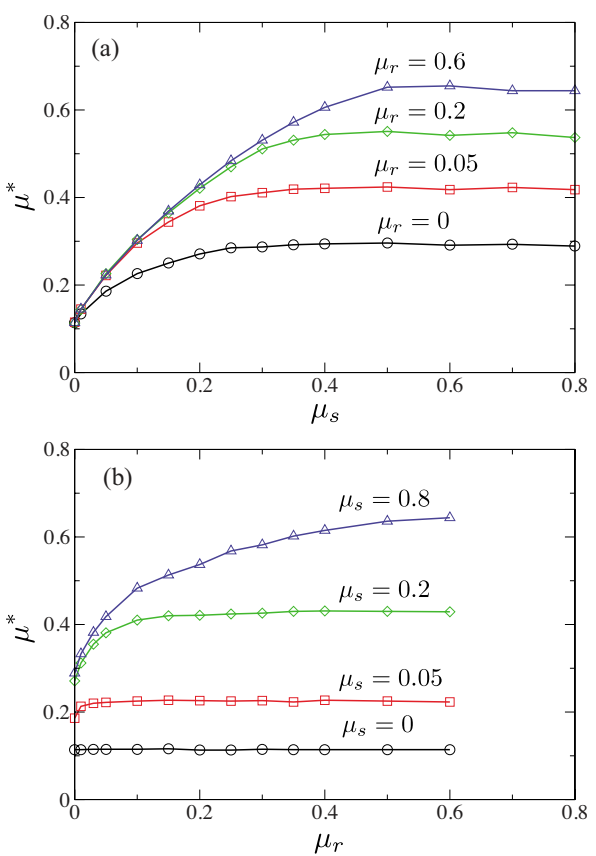

FIG. 7. (Color online) Internal friction coefficient $\mu^{*}$ as a function of $\mu_{s}$ for several fixed values of $\mu_{r}$ (a), and as a function of $\mu_{s}$ for several fixed values of $\mu_{r}$ (b).

This map reveals a rather simple structure with an approximate symmetry with respect to the line $\mu_{s}=\mu_{r}$. We observe again the saturation effect both with $\mu_{s}$ and $\mu_{r}$, so that two phases can clearly be distinguished on the contour map: (1) a sliding phase in which $\mu^{*}$ depends only on $\mu_{s}$, and (2) a rolling phase in which $\mu^{*}$ depends only on $\mu_{r}$. The transition zone between these two phases, marked by a gray stripe in Fig. 8 , is narrow $(\simeq 0.15)$.

This two-phase behavior suggests that the system organizes itself so as to favor the mode of relative motion at the contacts (sliding or rolling) for which the friction parameter is lower. This behavior can be elucidated by considering the populations of contacts depending on their rolling and sliding status. There are four categories: (1) sliding-nonrolling (s-nr), (2) nonsliding-rolling (ns-r), (3) sliding-rolling (s-r)

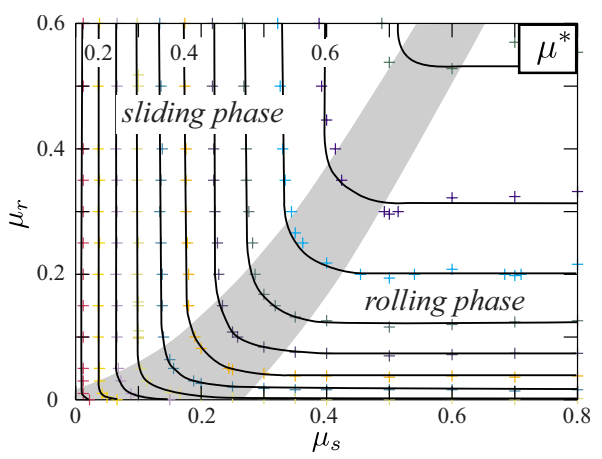

FIG. 8. (Color online) Contour map of the internal friction coefficient $\mu^{*}$ as a function of sliding and rolling friction coefficients $\mu_{s}$ and $\mu_{r}$. The gray stripe represents the transition zone between a phase where $\mu^{*}$ depends only on the coefficient of sliding friction $\mu_{s}$ and a phase where $\mu^{*}$ depends only on the coefficient of rolling friction $\mu_{r}$.
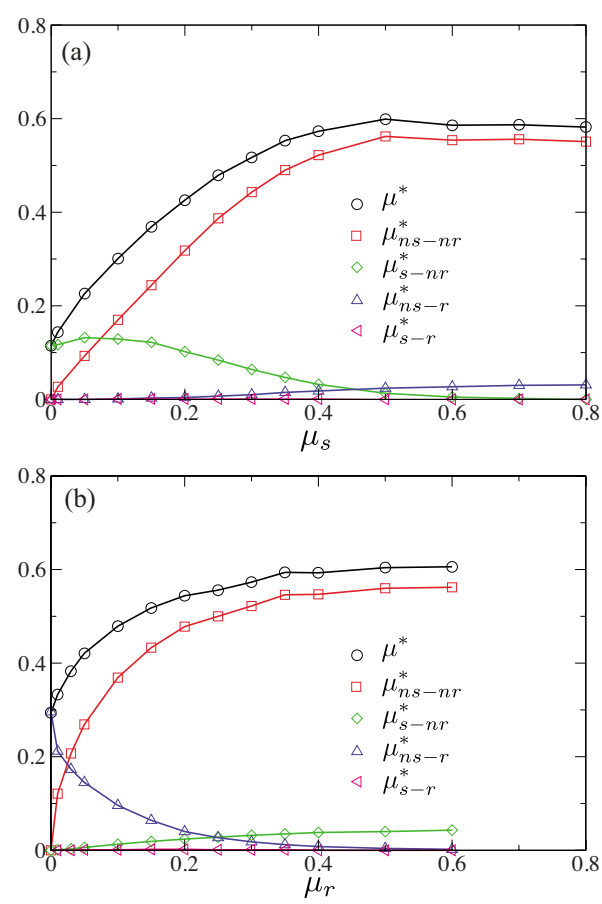

FIG. 9. (Color online) Partial friction coefficients as a function of $\mu_{s}$ for $\mu_{r}=0.3$ (a), and as a function of $\mu_{r}$ for $\mu_{s}=0.4$ (b).

and (4) nonsliding-nonrolling (ns-nr). The first three populations correspond to the "mobilized" contacts whereas the last population defines the "interlocked" contact network. The contribution of each population to the total shear stress can be calculated from the general expression of the stress tensor [i.e., Eq. (8)] by restricting the summation to the contacts belonging to that population. Hence, partial friction coefficients $\mu_{\mathrm{s}-\mathrm{nr}}^{*}, \mu_{\mathrm{ns}-\mathrm{r}}^{*}, \mu_{\mathrm{s}-\mathrm{r}}^{*}$, and $\mu_{\mathrm{ns}-\mathrm{nr}}^{*}$ can be defined for each population. This is an additive decomposition so that

$$
\mu^{*}=\mu_{\mathrm{s}-\mathrm{nr}}^{*}+\mu_{\mathrm{ns}-\mathrm{r}}^{*}+\mu_{\mathrm{s}-\mathrm{r}}^{*}+\mu_{\mathrm{ns}-\mathrm{nr}}^{*} .
$$

Figure 9 shows the evolution of the partial friction coefficients with $\mu_{s}$ for $\mu_{r}=0.3$, and with $\mu_{r}$ for $\mu_{s}=0.4$. The contribution $\mu_{\mathrm{s}-\mathrm{r}}^{*}$ of both sliding and rolling contacts is negligibly small. On the contrary, the contribution $\mu_{\mathrm{ns}-\mathrm{nr}}^{*}$ of interlocked contacts prevails except at low sliding or rolling coefficients $\left(\mu_{s}<0.1\right.$ and $\left.\mu_{r}<0.05\right)$ where the dominant contribution comes from sliding or rolling contacts, respectively. On the other hand, as $\mu_{s}$ increases, the contribution $\mu_{\mathrm{s}-\mathrm{nr}}^{*}$ of sliding-nonrolling contacts declines in favor of $\mu_{\mathrm{ns}-\mathrm{r}}^{*}$ which increases at the same time. Symmetrically, as $\mu_{r}$ increases, the contribution $\mu_{\text {ns-r }}^{*}$ of nonsliding-rolling contacts declines in favor of $\mu_{\mathrm{s}-\mathrm{nr}}^{*}$ which increases.

In order to characterize the dominant mode of relative motion at the contacts, we consider these two last populations and define the parameter $k$ by

$$
k=\frac{\mu_{\mathrm{s}-\mathrm{nr}}^{*}-\mu_{\mathrm{ns}-\mathrm{r}}^{*}}{\mu_{\mathrm{s}-\mathrm{nr}}^{*}+\mu_{\mathrm{ns}-\mathrm{r}}^{*}} .
$$

This parameter varies in the range $[-1,1]$ as a function of $\mu_{s}$ and $\mu_{r}$. The dominant mode is sliding if $k>0$ and rolling if 


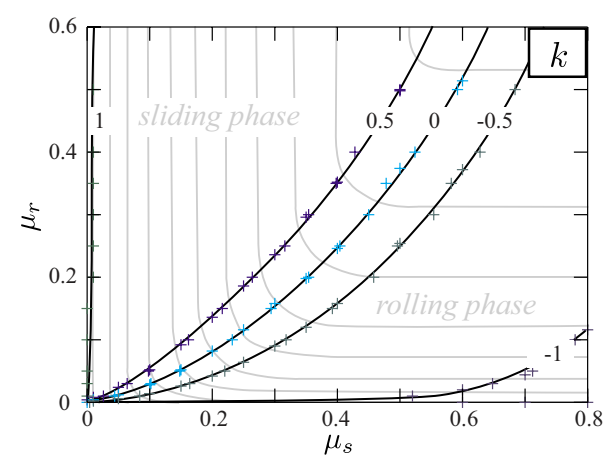

FIG. 10. (Color online) Contour map of the parameter $k$ (see text) as a function of the contact friction coefficients $\mu_{s}$ and $\mu_{r}$.

$k<0$. Figure 10 shows the contour map of $k$ as a function of $\mu_{s}$ and $\mu_{r}$ together with the contour lines of $\mu^{*}$. We see that the contour for $k=0$ matches closely the transition between the sliding phase and the rolling phase. In other words, the partial friction coefficients $\mu_{\mathrm{s}-\mathrm{nr}}^{*}$ and $\mu_{\mathrm{ns}-\mathrm{r}}^{*}$ reflect the effect of the contact friction coefficients $\mu_{s}$ and $\mu_{r}$ with respect to $\mu^{*}$.

\section{SOLID FRACTION AND COLUMN-LIKE STRUCTURES}

In this section, we study the packing structures in the steady state as a function of $\mu_{s}$ and $\mu_{r}$. The compactness of the structure can be represented by the solid fraction $\nu$ defined by

$$
\nu=\frac{V_{p}}{V},
$$

where $V_{p}$ is the total volume of the particles and $V$ is the total volume of the packing. Figure 11 shows the contour map of $\nu$ as a function of $\mu_{s}$ and $\mu_{r}$. We see that, as for $\mu^{*}$, at low values of $\mu_{s}$, the solid fraction $\nu$ saturates with increasing $\mu_{r}$. However, the behavior is different at higher values of $\mu_{s}$ and $\mu_{r}$, breaking thus the expected symmetry between the two phases.

In fact, in contrast to $\mu^{*}$, the solid fraction as defined by Eq. (12) comprises both load-carrying and floating particles. But the fraction of floating particles is expected to increase with $\mu_{s}$ and $\mu_{r}$ as a result of enhanced arching effect. We

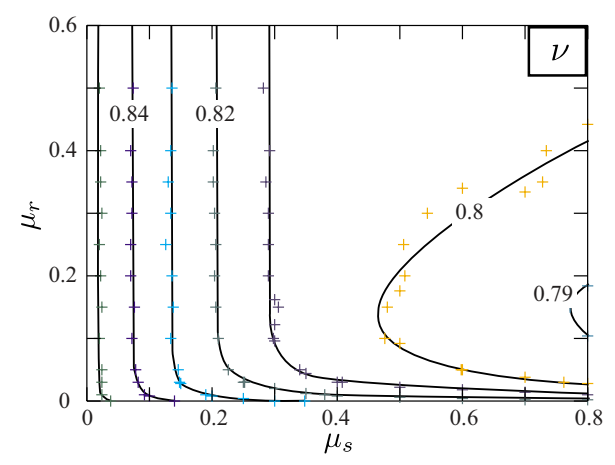

FIG. 11. (Color online) Contour map of the solid fraction $\nu$ as a function of $\mu_{s}$ and $\mu_{r}$.

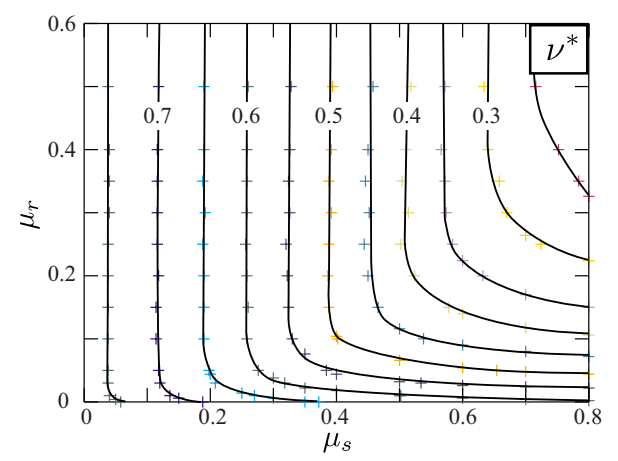

FIG. 12. (Color online) Contour map of the solid fraction $\nu^{*}$ of the load-carrying particles as a function of $\mu_{s}$ and $\mu_{r}$.

thus consider the solid fraction $\nu^{*}$ of load-carrying particles defined by

$$
\nu^{*}=\frac{V_{p}^{*}}{V},
$$

where $V_{p}^{*}$ is the volume of the load-carrying particles. Figure 12 shows the contour map of $\nu^{*}$ as a function of $\mu_{s}$ and $\mu_{r}$. Interestingly, the global aspect of this map is now symmetric with respect to the two friction parameters. By comparison with Fig. 8, we see that the highest levels of shear strength correspond to the least compact structures. The reason is that steady state shearing implies larger free volumes accessible to the particles when the relative degrees of freedom between particles are restrained due to higher rolling or sliding contact friction. Remark that the solid fraction $\nu^{*}$ can be as small as 0.3 and even lower (see the contour map in Fig. 12).

Low values of solid fraction $\nu^{*}$ coincide with the formation of column-like structures, i.e. long chains of particles with only two contacts. Figure 13 shows snapshots of the load-carrying contact network and normal forces in two packings denoted $S 1$ and $S 2$. The coefficients of friction are $\mu_{s}=0.4$ and $\mu_{r}=0$ in $S 1$, and $\mu_{s}=0.7$ and $\mu_{r}=0.5$ in $S 2$. We observe two important differences between the load-carrying structures in these packings. (1) In packing $S 1$ almost all particles belong to the load-carrying network, whereas in $S 2$ most particles are floating and the load-carrying particles form columns. (2) In packing $S 1$ the contacts carrying large forces (strong contacts) form chainlike structures propped by contacts carrying small forces (weak contacts). In contrast, in packing $S 2$, the force chains are not propped everywhere. This aspect is analyzed in more detail in the following section.

\section{STRESS TRANSMISSION}

\section{A. Probability density functions}

In close correlation with shear strength and solid fraction, the stress transmission is strongly influenced by rolling and sliding friction. Figure 14 displays the probability density functions $P_{n}$ of normal forces $f_{n}$ in the packings $S 1$ and $S 2$. The data are cumulated from several snapshots in the steady state, corresponding to a total number of $1.3 \times 10^{6}$ contact forces for packing $S 1$ and of $0.4 \times 10^{6}$ contact forces for 

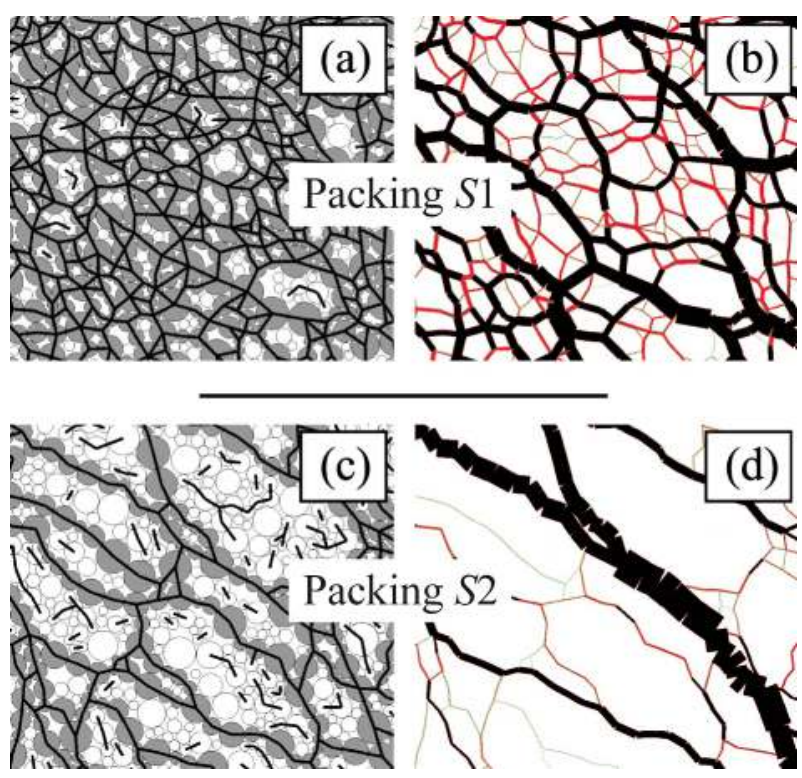

FIG. 13. (Color online) Snapshots of (a) the load-carrying network in packing $S 1$, (b) the normal forces in packing $S 1$, (c) the load-carrying network in packing $S 2$, and (d) the normal forces in packing $S 2$. In (a) and (c) the particles that participate in the loadcarrying network are in gray, and the black lines represent the contact network. In (b) and (d) the black lines represent strong forces $\left(f_{n}\right\rangle\left\langle f_{n}\right\rangle$, where $\left\langle f_{n}\right\rangle$ is the mean normal force) and red (gray) lines represent weak forces $\left(f_{n}<\left\langle f_{n}\right\rangle\right)$. Line thickness is proportional to the force magnitude.

packing $S 2$. As often observed in granular media [34,35], we find that the function $P_{n}$ is characterized by an exponential falloff for the forces above the average force $\left\langle f_{n}\right\rangle$ and a power-law distribution for the forces below $\left\langle f_{n}\right\rangle$ :
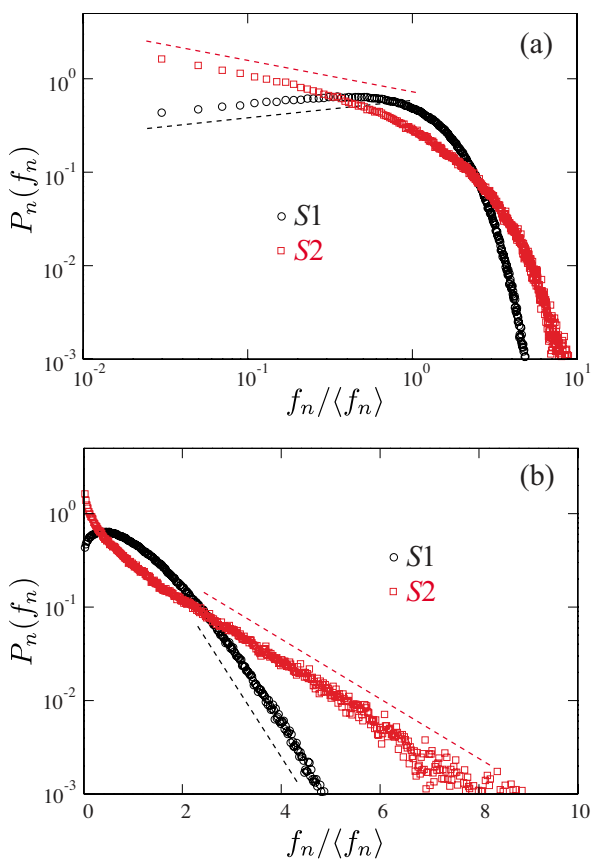

FIG. 14. (Color online) Probability density functions $P_{n}$ of normal forces $f_{n}$ in the packings $S 1$ and $S 2$ in $\log$-log (a) and log-linear (b) scales. The dashed lines are guides to the eyes.
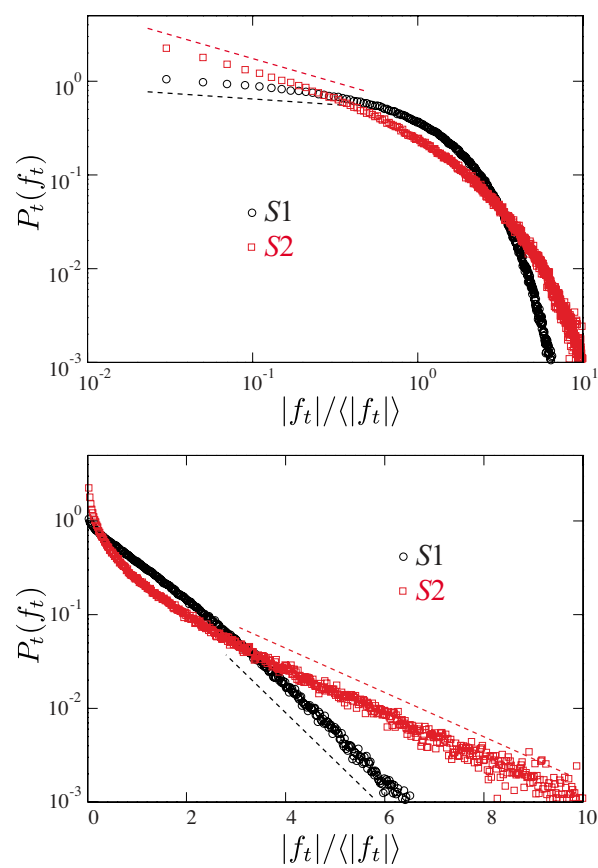

FIG. 15. (Color online) Probability density functions $P_{t}$ of tangential forces $f_{t}$ in the packings $S 1$ and $S 2$ in $\log -\log$ (a) and $\log$ linear (b) scales. The dashed lines are guides to the eyes.

$$
P_{n}\left(f_{n}\right) \propto \begin{cases}\left(\frac{f_{n}}{\left\langle f_{n}\right\rangle}\right)^{-\alpha_{n}}, & f_{n}<\left\langle f_{n}\right\rangle, \\ e^{\beta_{n}\left[1-f_{n} /\left\langle f_{n}\right\rangle\right]}, & f_{n}>\left\langle f_{n}\right\rangle,\end{cases}
$$

with $\alpha_{n} \simeq-0.17$ and $\beta_{n} \simeq 1.8$ in $S 1$, and $\alpha_{n} \simeq 0.37$ and $\beta_{n}$ $\simeq 0.81$ in $S 2$. Hence, the normal force distribution $P_{n}$ in packing $S 2$ (with $\mu_{r}=0.5$ ) is considerably broader in the range of strong forces, and the fraction of weak forces diverges as $f_{n} \rightarrow 0$.

Figure 15 shows the probability density function $P_{t}$ of friction forces $f_{t}$, which is also characterized by an exponential falloff for the forces above the average force $\left\langle\left|f_{t}\right|\right\rangle$ and a power-law distribution for the forces below $\left\langle\left|f_{t}\right|\right\rangle$ :

$$
P_{t}\left(f_{t}\right) \propto \begin{cases}\left(\frac{\left|f_{t}\right|}{\left\langle\left|f_{t}\right|\right\rangle}\right)^{-\alpha_{t}}, & \left|f_{t}\right|<\left\langle\left|f_{t}\right|\right\rangle, \\ e^{\left.\beta_{t}\left[1-\left|f_{t}\right| /\left|f_{t}\right|\right\rangle\right]}, & \left|f_{t}\right|>\left\langle\left|f_{t}\right|\right\rangle,\end{cases}
$$

with $\alpha_{t} \simeq 0.17$ and $\beta_{t} \simeq 1.1$ in $S 1$, and $\alpha_{t} \simeq 0.52$ and $\beta_{t}$ $\simeq 0.57$ in $S 2$.

The probability density function $P_{M}$ of contact torques is defined only in the packing $S 2$ with nonzero rolling resistance. As shown in Fig. 16, it can be also be described by

$$
P_{M}(M) \propto \begin{cases}\left(\frac{|M|}{\langle|M|\rangle}\right)^{-\alpha_{M}}, & |M|<\langle|M|\rangle, \\ e^{\left.\beta_{M}[1-|M| /|M|\rangle\right]}, & |M|>\langle|M|\rangle,\end{cases}
$$

with $\alpha_{M} \simeq 0.66$ and $\beta_{M} \simeq 0.49$.

These distributions show clearly the larger inhomogeneity of stress transmission in a granular packing in the presence of rolling resistance. A systematic investigation of force distributions will be reported elsewhere [36]. In addition, Figs. 

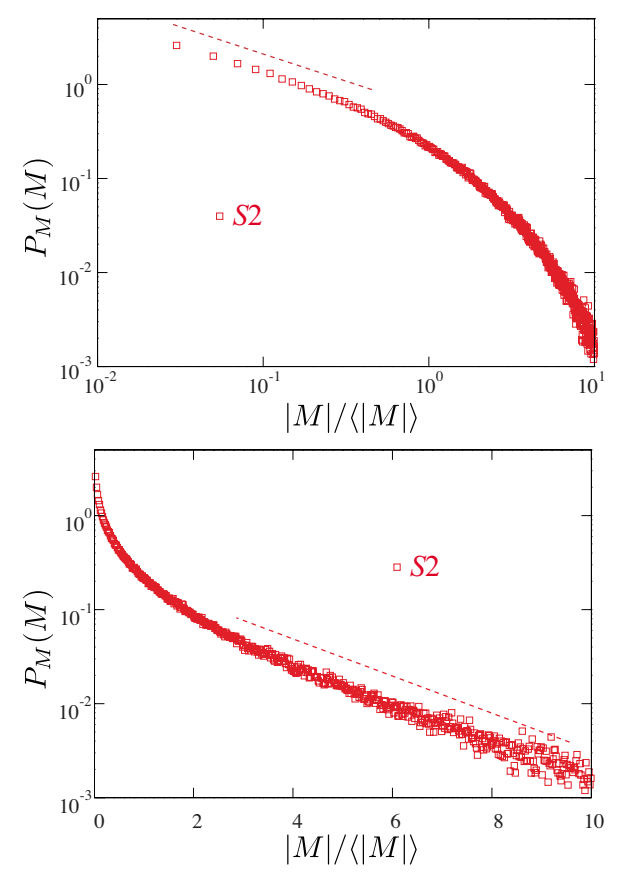

FIG. 16. (Color online) Probability density function $P_{M}$ of contact torques $M$ in the packing $S 2$ in $\log -\log$ (a) and log-linear (b) scales. The dashed lines are guides to the eyes.

14-16 show that, independently of the friction coefficients, the contacts can be classified in two sets characterized by a different distribution of contact forces. We will analyze this bimodal character of the contact network in the next section.

\section{B. Strong and weak force networks}

As mentioned in the previous section, it is a well-known feature of sheared granular media that the contacts belong to two coexisting networks termed strong and weak networks following Radjai et al. [37]. Moreover, these two networks are known to have different topologies and play different mechanical roles in the system. The contacts in the strong network correspond to chainlike structures distinctly observed in photoelastic images and called force chains; their function is to sustain the deviatoric load imposed on the system. The weak network is less visible, but its contacts are more important in number and have the essential function of propping the force chains. This property can be evidenced by considering the anisotropy of contact orientations. The strong contacts are oriented mainly along the major principal stress direction whereas the weak contacts are, on average, perpendicular. The average normal force $\left\langle f_{n}\right\rangle$ is generally found to be the approximate characteristic force differentiating between the two types of contacts. Here, we would like to study the effect of rolling friction on the nature of these two networks.

Let us consider the distribution $P_{\theta}(\theta)$ of contact orientations $\theta \in[0, \pi]$. Fig. 17 displays $P_{\theta}$ in polar coordinates for strong and weak contacts in packings $S 1$ with $\mu_{s}=0.4$ and $\mu_{r}=0$, and $S 2$ with $\mu_{s}=0.7$ and $\mu_{r}=0.5$. We see that in the packing $S 1$, where there is no rolling resistance, the strong network is indeed more anisotropic than the weak network
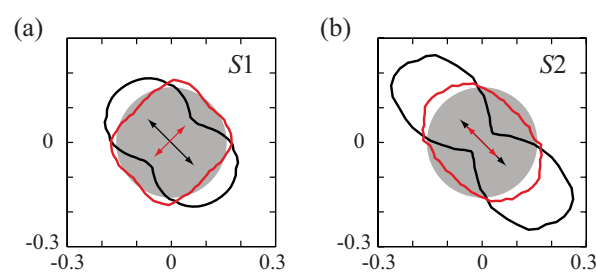

FIG. 17. (Color online) $P_{\theta}$ in polar coordinates for strong (black line) and weak [red (gray) line] contacts in packings $S 1$ with $\mu_{s}$ $=0.4$ and $\mu_{r}=0$ (a), and $S 2$ with $\mu_{s}=0.7$ and $\mu_{r}=0.5$ (b). The gray circle represents a reference isotropic distribution.

and the privileged directions of the two networks (longer axis of each diagram) are orthogonal. In contrast, in packing $S 2$, where both rolling and sliding friction coefficients are high, the privileged directions of the two populations are similar and coincide with the major principal stress direction $\theta_{\sigma}=3 \pi / 4$. This observation suggests that the function of the weak contacts in the packing $S 2$ is no longer to prop the force chains laterally, implying that the nature of the weak network is fundamentally different in the two packings. In the following, we analyze this point in terms of the anisotropies of the distributions of contact orientations.

The anisotropy of the distributions can be calculated by expanding $P_{\theta}$ on the Fourier basis. Since $P_{\theta}$ is $\pi$ periodic, we have

$$
P(\theta)=\frac{1}{\pi}\left[1+a \cos 2\left(\theta-\theta_{a}\right)+b \cos 4\left(\theta-\theta_{b}\right)+\cdots\right],
$$

where the coefficients $a, b, \ldots$ are the anisotropy parameters of increasing order and the angles $\theta_{a}, \theta_{b}, \ldots$ are the phases.

Generally the first-order anisotropy $a$ captures the main trend. It can be calculated from the lowest-order fabric tensor $\boldsymbol{F}$ defined by

$$
F_{\alpha \beta}=\frac{1}{N_{c} \in V} \sum_{\alpha} n_{\alpha}^{c} n_{\beta}^{c}
$$

where $\alpha$ and $\beta$ represent the components in an orthonormal reference frame, $\boldsymbol{n}^{c}$ is the normal unit vector at contact $c$, and the summation runs over all contacts $c$ in the control volume $V$. It is easily shown that

$$
a=2\left(F_{1}-F_{2}\right),
$$

where $F_{1}$ and $F_{2}$ are the principal values of $\boldsymbol{F}$. By definition, the anisotropy $a$ is zero or positive. For the analysis of the anisotropy in strong and weak networks, or in subsets of contacts, where the directions $\theta_{a}$ are not similar, it is more convenient to introduce the phase factor by multiplying $a$ by $\cos 2\left(\theta_{a}-\theta_{\sigma}\right)$, where $\theta_{\sigma}$ is the direction of the major principal stress. Hence, we define the "signed" anisotropy $a^{\prime}$ by

$$
a^{\prime}=2\left(F_{1}-F_{2}\right) \cos 2\left(\theta_{a}-\theta_{\sigma}\right) .
$$

This anisotropy is positive when the two major principal directions are in phase, i.e., when $\left|\theta_{a}-\theta_{\sigma}\right|<\pi / 4$, otherwise the anisotropy is negative. In our simple shear tests, we have $\theta_{\sigma}=3 \pi / 4$. 


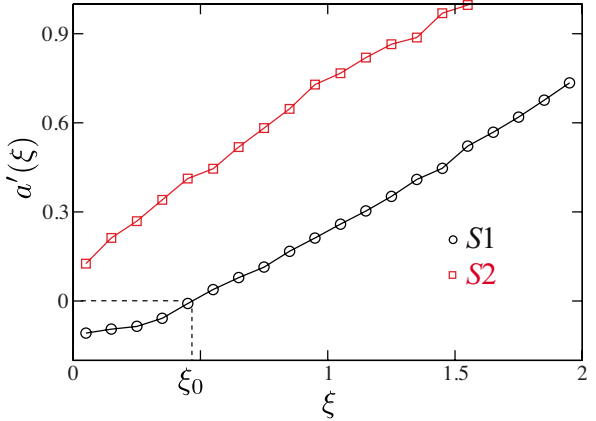

FIG. 18. (Color online) Signed anisotropy $a^{\prime}$ as a function of the force level $\xi$ in packings $S 1$ with $\mu_{s}=0.4$ and $\mu_{r}=0$, and $S 2$ with $\mu_{s}=0.7$ and $\mu_{r}=0.5$.

In order to analyze the anisotropy of force networks, we consider the subsets $\mathcal{S}(\xi)$ of contacts carrying a normal force level $\xi=f_{n} /\left\langle f_{n}\right\rangle \in[\xi-\Delta \xi, \xi+\Delta \xi]$ so that each contact belongs to only one subset. The weak and strong networks correspond to $\cup_{\xi<1} \mathcal{S}(\xi)$ and $\cup_{\xi>1} \mathcal{S}(\xi)$, respectively. The anisotropy $a^{\prime}(\xi)$ of each set $\mathcal{S}(\xi)$ can be calculated from the definition of the fabric tensor by restricting the summation to the contacts belonging to this set:

$$
F_{\alpha \beta}(\xi)=\frac{1}{N_{c}(\xi)} \sum_{c \in \mathcal{S}(\xi)} n_{\alpha}^{c} n_{\beta}^{c},
$$

where $N_{c}^{\xi}$ is the cardinal of the set $\mathcal{S}(\xi)$.

Figure 18 shows $a^{\prime}(\xi)$ in packings $S 1$ with $\mu_{s}=0.4$ and $\mu_{r}=0$, and $S 2$ with $\mu_{s}=0.7$ and $\mu_{r}=0.5$. We see that in the packing $S 1$, where there is no rolling resistance, the anisotropies at weak force levels are negative (corresponding to a privileged orientation along the minor principal stress direction) up to a point $\xi_{0}$ where $a^{\prime}(\xi)$ changes sign and becomes positive. In contrast, in the packing $S 2$, where there is rolling resistance, the anisotropies at both weak and strong force levels are positive, showing that, on average, the privileged orientation of all subsets of contacts is the same. This shows that all the contacts in the weak network do not play the same role in the system. Rather, the propping role seems to be a property of a subnetwork of contacts inside the weak network carrying forces below a characteristic value $\xi_{0}\left\langle f_{n}\right\rangle$. Interestingly, this "propping network" of contacts is completely absent in the limit of high rolling resistance and rolling friction (i.e., in packing $S 2$ ).

To push this analysis further, let us consider the contribution $\mu^{*}(\xi)$ of each subset $\mathcal{S}(\xi)$ of contacts to the friction coefficient $\mu^{*}$, which is given by

$$
\mu^{*}(\xi)=\frac{\tau(\xi)}{\sigma},
$$

where $\tau(\xi)$ is the tangential stress calculated from the definition of the stress tensor by restricting the summation to the contacts belonging to $\mathcal{S}(\xi)$ and $\sigma$ is the normal stress when considering all contacts. Figure 19 shows $\mu(\xi)$ in packings $S 1$ and $S 2$. We see that in the packing $S 1$, where there is no rolling resistance, the contribution of the contacts with force levels $\xi<\xi_{0}$ to the strength of the packing is nearly zero, and

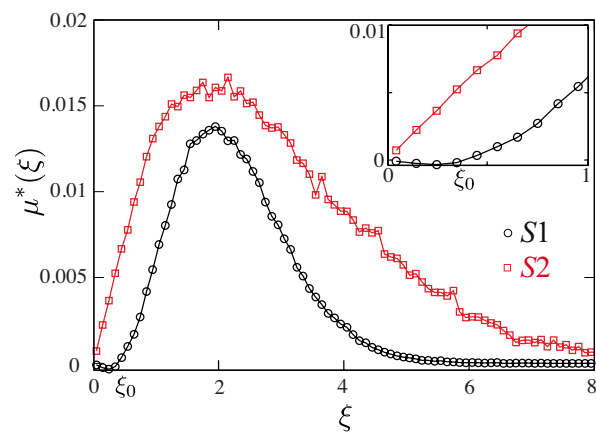

FIG. 19. (Color online) Contribution to the coefficient of friction $\mu^{*}$ as a function of the force level $\xi$ in packings $S 1$ with $\mu_{s}$ $=0.4$ and $\mu_{r}=0$, and $S 2$ with $\mu_{s}=0.7$ and $\mu_{r}=0.5$.

the contribution of the contacts with force levels $\xi>\xi_{0}$ to $\mu^{*}$ is positive. This means that the deviatoric load applied to the system is totally sustained by the contacts carrying forces above $\xi_{0}\left\langle f_{n}\right\rangle$. In contrast, in the packing $S 2$, where there is rolling resistance, the contribution of all contacts, regardless of their force level, is positive, which means that, in this packing, the function of the weak network is also to sustain the deviatoric load applied to the system.

Figure 20 shows the contour map of the characteristic force level $\xi_{0}$ as a function of $\mu_{s}$ and $\mu_{r}$. As $\mu_{s}$ and $\mu_{r}$ increase, $\xi_{0}$ decreases and even vanishes for large values of friction coefficients. This is consistent with the observation that the force chains are gradually replaced by columns of particles which do not need to be propped. However, the total vanishing of the propping network (i.e., $\xi_{0}=0$ ) takes place at $\mu_{s}=0.6$ and $\mu_{r}=0.25$, and thus it cannot occur without rolling friction. This transition reflects a qualitative change in the condition of local force and torque balance in the presence of interlocking.

\section{SUMMARY AND DISCUSSION}

In summary, by means of contact dynamics simulations, we investigated a class of granular material involving interlocking actions between the particles. Interlocking was modeled as a rolling friction law relating the contact torque to the relative rotation between two particles with a rolling friction coefficient $\mu_{r}$ analogous to the sliding friction coefficient $\mu_{s}$.

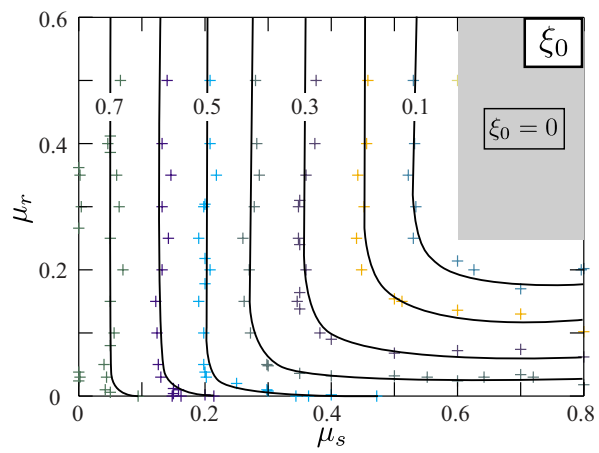

FIG. 20. (Color online) Contour map of $\xi_{0}$ (see text) as a function of $\mu_{s}$ and $\mu_{r}$. 
A large number of 2D polydisperse numerical samples were sheared for a broad range of friction parameters $\mu_{s}$ et $\mu_{r}$ and the steady state was analyzed in terms of shear strength, fabric properties, and force transmission.

We found an interesting symmetry between the effects of $\mu_{s}$ and $\mu_{r}$ on the macroscopic response of the packing. In particular, the coefficient of internal friction $\mu^{*}$ saturates as a function of $\mu_{s}$ with a saturation value that increases with $\mu_{r}$, and, symmetrically, it saturates as a function of $\mu_{r}$ with a saturation value that increases with $\mu_{s}$. As a result, two distinct phases can be distinguished in which $\mu^{*}$ is controlled by either $\mu_{s}$ or $\mu_{r}$. This suggests that the dominant mode of relative motion at the contacts (sliding or rolling) is the one which minimizes the coefficient of internal friction. This behavior was shown to be consistent with the relative contributions of sliding and rolling contacts to $\mu^{*}$.

These findings are in agreement with those reported in $[7,20,38]$, stating that contact rolling plays a major role in the shear strength of granular media. Nevertheless, our results show quantitatively when contact rolling prevails. Contact rolling is the dominant mode when $\mu_{s}$ is large compared to $\mu_{r}$, but contact sliding is the dominant mode when $\mu_{r}$ is large compared to $\mu_{s}$. The reason is that high values of one of the two friction coefficients tend to block the corresponding degree of freedom enhancing thus the alternative mode of relative motion at the contacts.

We also found that the combined effect of sliding friction and interlocking strongly affects the microstructure of the packing. In particular, high values of $\mu_{s}$ and $\mu_{r}$ enhance the arching effect, causing the force carrying backbone to take an increasingly columnar aspect. The formation of these columns affects also the mechanical role played by the weak network. A transition occurs for particular values of $\mu_{s}$ and $\mu_{r}$ to a phase where the fraction of the weak network that props the force chains (i.e., the propping network) is completely absent $\left(\xi_{0}=0\right)$, and both networks, weak and strong, contribute to sustain the deviatoric load applied to the system. In this phase, columns are the dominant force transmitting structure, and, as a consequence of an enhanced arching effect, the solid fraction of the load-carrying backbone can be extremely low, e.g., 0.3 .
We also presented the probability distributions of contact forces and torques. These distributions were shown to follow a power law in the range of weak forces and fall off exponentially for strong forces. Another important point is that the vanishing of the propping network does not imply a decrease in the fraction of contacts carrying weak forces. Actually, the proportion of weak contacts increases, even if their mechanical role evolves with friction coefficients. This is a consequence of significant changes in the local equilibrium conditions as the contacts are allowed to exert torques.

It is evident that the complex interactions between particles in real granular materials cannot be fully captured by a simple model of circular particles with sliding and rolling friction at their contacts. Nevertheless, we think that some of the trends presented in this paper, such as the occurrence of a dominant mode at the contacts, the formation of new structures of force transmission and the changes in the mechanical role of the weak network, are robust features of granular media involving rolling resistance. We believe that these findings are relevant for a better understanding of the role played by the local thresholds on the rich behavior of sheared granular materials.

An important issue not tackled in this paper concerns the effect of rolling resistance with respect to particle displacements and rotations between successive equilibrium states. Clearly, as shown in Fig. 5, the particle motions are increasingly fluctuating as rolling friction is higher. This shows that the transition is increasingly dynamic due to the breakdown of the supporting structures shown in Fig. 13. These kinematic aspects involving the formation and breakdown of local rigid structures, spatial correlations of nonaffine particle velocities, dynamic rearrangements, and dissipation of kinetic energy are presently under investigation.

\section{ACKNOWLEDGMENTS}

We warmly thank E. Azéma for useful and stimulating discussions, and J. Tack for technical support. This work was supported by the project Ecos Nord No. C08U01.
[1] D. M. Wood, Soil Behaviour and Critical State Soil Mechanics (Cambridge University Press, Cambridge, England, 1990).

[2] J. K. Mitchell and K. Soga, Fundamentals of Soil Behavior, 3rd ed. (Wiley, New York, 2005).

[3] A. Caquot, Équilibre des Massifs à Frottement InterneStabilité des Terres Pulvérulentes ou Cohérentes (GauthierVillars, Paris, 1934).

[4] A. W. Bishop, Geotechnique 4, 43 (1954).

[5] P. W. Rowe, Proc. R. Soc. London, Ser. A 269, 500 (1962).

[6] A. E. Skinner, Geotechnique 19, 150 (1969).

[7] M. Oda, J. Konishi, and S. Nemat-Nasser, Mech. Mater. 1, 269 (1982).

[8] F. Radjai, D. E. Wolf, S. Roux, M. Jean, and J. J. Moreau, in Powders and Grains 97, edited by R. P. Behringer and J. T. Jenkins (Balkema, Rotterdam, 1997), pp. 211-214.
[9] F. Radjai, D. E. Wolf, M. Jean, and J. J. Moreau, Phys. Rev. Lett. 80, 61 (1998).

[10] K. Iwashita and M. Oda, J. Eng. Mech. 124, 285 (1998).

[11] Y. C. Zhou, B. D. Wright, R. Y. Yang, B. H. Xu, and A. B. Yu, Physica A 269, 536 (1999).

[12] J.-Y. Delenne, M. S. El Youssoufi, F. Cherblanc, and J.-C. Bénet, Int. J. Numer. Analyt. Meth. Geomech. 28, 1577 (2004).

[13] I. Bratberg, F. Radjai, and A. Hansen, Phys. Rev. E 66, 031303 (2002).

[14] D. Kadau, G. Bartels, L. Brendel, and D. E. Wolf, Comput. Phys. Commun. 147, 190 (2002).

[15] M. J. Jiang, H. S. Yu, and D. Harris, Int. J. Numer. Analyt. Meth. Geomech. 30, 723 (2006).

[16] F. A. Gilabert, J.-N. Roux, and A. Castellanos, Phys. Rev. E 75, 011303 (2007). 
[17] S. Latham, S. Abe, and P. Mora, in Powders and Grains, Fifth International Conference on Micromechanics of Granular Media, Stuttgart, edited by H. H. R. García Rojo and S. McNamara (A. A. Balkema, Rotterdam, 2005), Vol. 1, pp. 213-217.

[18] F. Calvetti and R. Nova, in Powders and Grains, Fifth International Conference on Micromechanics of Granular Media, Stuttgart (Ref. [17]), Vol. 1, pp. 245-249.

[19] F. Calvetti, R. Nova, and S. Utili, in Powders and Grains, Fifth International Conference on Micromechanics of Granular Media, Stuttgart (Ref. [17]), Vol. 1, pp. 671-675.

[20] F. Alonso-Marroquín, I. Vardoulakis, H. J. Herrmann, D. Weatherley, and P. Mora, Phys. Rev. E 74, 031306 (2006).

[21] J. J. Moreau, Eur. J. Mech. A/Solids 13 (Suppl.), 93 (1994).

[22] M. Jean, Mechanics of Geometrical Interfaces (Elsevier, New York, 1995), pp. 463-486.

[23] M. Jean, Comput. Methods Appl. Mech. Eng. 117, 235 (1999).

[24] M. Jean, Micromécanique des Matériaux Granulaires (Hermes, Paris, 2001), pp. 199-324.

[25] F. Radjaï, in Physics of Dry Granular Media, edited by H. J. Herrmann, J. P. Hovi, and S. Luding (Kluwer Academic Publishers, Dordrecht, 1998), pp. 305-312.
[26] A. Taboada, K.-J. Chang, F. Radjai, and F. Bouchette, J. Geophys. Res. 110, B09202 (2005).

[27] M. Renouf, F. Dubois, and P. Alart, J. Comput. Appl. Math. 168, 375 (2004).

[28] C. Voivret, F. Radjaï, J.-Y. Delenne, and M. S. El Youssoufi, Phys. Rev. E 76, 021301 (2007).

[29] G. MiDi, Eur. Phys. J. E 14, 341 (2004).

[30] F. da Cruz, S. Emam, M. Prochnow, J.-N. Roux, and F. Chevoir, Phys. Rev. E 72, 021309 (2005).

[31] I. Agnolin and J.-N. Roux, Phys. Rev. E 76, 061303 (2007).

[32] J. J. Moreau, in Friction, Arching, Contact Dynamics (World Scientific, Singapore, 1997), pp. 233-247.

[33] L. Staron, F. Radjaï, and J.-P. Vilotte, Eur. Phys. J. E 18, 311 (2005).

[34] F. Radjai, M. Jean, J.-J. Moreau, and S. Roux, Phys. Rev. Lett. 77, 274 (1996).

[35] D. M. Mueth, H. M. Jaeger, and S. R. Nagel, Phys. Rev. E 57, 3164 (1998).

[36] N. Estrada, A. Taboada, and F. Radjaï (unpublished).

[37] F. Radjaï, D. E. Wolf, M. Jean, and J. J. Moreau, Phys. Rev. Lett. 80, 61 (1998).

[38] J. P. Bardet, Mech. Mater. 18, 159 (1994). 